\title{
Aspectos éticos para a enfermagem em cuidados paliativos na terapia intensiva: uma
}

\section{revisão integrativa}

\author{
Ethical aspects for palliative care nursing in intensive care: an integrative review \\ Aspectos éticos de la enfermería de cuidados paliativos en cuidados intensivos: una revisión
}

integrativa

Recebido: 28/11/2021 | Revisado: 03/12/2021 | Aceito: 04/12/2021 | Publicado: 14/12/2021

\author{
Adriana Maria de Oliveira \\ ORCID: https://orcid.org/0000-0002-9115-5803 \\ Universidade Federal do Estado do Rio de Janeiro, Brasil \\ E-mail: dicaoliveira.1177@gmail.com \\ Paulo Sérgio Marcellini \\ ORCID: https://orcid.org/0000-0001-5618-5348 \\ Universidade Federal do Estado do Rio de Janeiro, Brasil \\ E-mail: marcellinips@yahoo.com
}

\begin{abstract}
Resumo
Objetivos: realizar uma revisão integrativa das produções científicas sobre os aspectos éticos dos cuidados paliativos para a enfermagem na terapia intensiva. Metodologia: Revisão Integrativa da Literatura (RIL), exploratória, qualitativa. A escolha da RIL ocorreu diante da busca de informações sobre o tema, a partir de estudos já publicados. Resultados: Foram identificados 1035 artigos nas bases de dados Pubmed, Embase, BVS/Lilacs e Cinahl. Destes, foram eliminados 73 artigos duplicados com e sem o descritor UTI, totalizando 962 artigos. Ao desconsiderar o descritor "terapia intensiva" foram encontrados 849 artigos e ao incluir o descritor "terapia intensiva", foram achados 113 artigos. Consideramos os artigos com resumo completo nos idiomas inglês, português e espanhol com o descritor UTI, resultando em 113 artigos. Destes, 9 foram selecionados. Foram excluídos 104, sendo 98 por fugirem do seu escopo e 6 por não apresentarem resumo completo, apesar da tentativa de acesso do manuscrito completo. Conclusão: É urgente pensarmos e repensarmos nossas condutas diante do paciente e traçarmos estratégias para a abordagem e prática dos cuidados paliativos em UTI, haja vista a importância da introdução deste cuidado tão essencial desde o diagnóstico. Não obstante ao progresso das produções científicas sobre questões éticas deste cuidado é de suma importância para que a temática seja amplamente discutida entre os profissionais implicados e ao se depararem com o paciente em cuidado paliativo na terapia intensiva possa ter condutas mais assertivas e comunicação mais inclusivoparticipativa de todos os profissionais na tomada de decisões.
\end{abstract}

Palavras-chave: Ética; Enfermagem; Cuidados paliativos; Terapia intensiva.

\begin{abstract}
Objectives: Conducting an integrative review of scientific productions on the ethical aspects of palliative care for nursing in intensive care. Methodology: An Integrative Literature Review (ILR), exploratory, qualitative. The choice of ILR occurred in the face of the search for information on the subject, based on studies already published. Results: A total of 1035 articles were identified in the PubMed, Embase, BVS//Lilacs and Cinahl databases. Of these, 73 duplicate articles were eliminated with and without the ICU descriptor, totaling 962 articles. When disregarding the descriptor "intensive care", 849 articles were found and, by including the descriptor "intensive care", 113 articles were found. We considered the articles with complete abstract in English, Portuguese and Spanish with the descriptor ICU, resulting in 113 articles. Of these, 9 were selected. A hundred were excluded, 98 for fleeing its scope and 6 for not presenting a complete abstract, despite the attempt to access the complete manuscript. Conclusion: It is urgent to think and rethink our conducts before the patient and outline strategies for the approach and practice of palliative care in ICU, given the importance of introducing this care so essential since diagnosis. Despite the progress of scientific productions on ethical issues of this care, it is of paramount importance for the theme to be widely discussed among the professionals involved and when they come across the patient in palliative care in intensive care, he may have more assertive conducts and more inclusive-participatory communication of all professionals in decision-making.
\end{abstract}

Keywords: Ethics; Nursing; Palliative care; Intensive therapy.

\section{Resumen}

Objetivos: Realizar una revisión integradora de las producciones científicas sobre los aspectos éticos de los cuidados paliativos para la enfermería en cuidados intensivos. Metodología: Revisión Integradora de la Literatura (RIL), exploratoria, cualitativa. La elección de RIL se produjo ante la búsqueda de información acerca del tema, en base a 
estudios ya publicados. Resultados: Se identificaron un total de 1035 artículos en las bases de datos PubMed, Embase, BVS/Lilacs y Cinahl. De estos, 73 artículos duplicados fueron eliminados con y sin el descriptor de UCI, totalizando 962 artículos. Al desconocer el descriptor "cuidados intensivos", se encontraron 849 artículos y, al incluir el descriptor "cuidados intensivos", se encontraron 113 artículos. Se consideraron los artículos con resumen completo en inglés, portugués y español con el descriptor UCI, resultando en 113 artículos. De estos, 9 fueron seleccionados. Cien centenares fueron excluidos, 98 por huir de su alcance y 6 por no presentar un resumen completo, a pesar del intento de acceder al manuscrito completo. Conclusión: Es urgente pensar y repensar nuestras conductas ante el paciente y esbozar estrategias para el abordaje y la práctica de los cuidados paliativos en UCI, dada la importancia de introducir estos cuidados tan esenciales desde el diagnóstico. A pesar del avance de las producciones científicas acerca de cuestiones éticas de esta atención, es de suma importancia que el tema sea ampliamente discutido entre los profesionales involucrados y cuando se encuentran con el paciente en cuidados paliativos en cuidados intensivos, puede tener conductas más asertivas y una comunicación más inclusiva-participativa de todos los profesionales en la toma de decisiones.

Palabras clave: Ética; Enfermería; Cuidados paliativos; Terapia intensiva.

\section{Introdução}

Unidade de Terapia Intensiva (UTI) é considerada área crítica destinada à internação de pacientes graves, que requerem atenção profissional especializada de forma contínua, materiais específicos e tecnologias necessárias ao diagnóstico, monitorização e terapia (CFM, 2020). Essa assistência é prestada de forma contínua 24 horas por dia por equipe multidisciplinar especializada (Brasil, 2020). Como consequência disso é um setor que reúne diversos aparatos tecnológicos e essenciais para a manutenção dos pacientes que ali se encontram.

Os pacientes que necessitam de internação nessa unidade apresentam dentre outras causas às doenças infecciosas, a angina instável, o infarto agudo do miocárdio, a insuficiência respiratória aguda, o edema agudo de pulmão e outras comorbidades onde cada perfil é direcionado ao tipo de UTI onde deve receber o cuidado, uma vez que têm se tornado cada vez mais específicas, tais como a UTI cardiológica, de queimados, doentes crônicos, pós-operatórios e oncológicas (Cavalcanti et al., 2019).

As UTIs oncológicas têm expandido seus serviços pelo crescimento exponencial de novos casos de câncer, que é considerado como o principal problema de saúde pública no mundo e já está entre as quatro principais causas de morte prematura (antes dos 70 anos de idade) na maioria dos países. A mais recente estimativa mundial, ano 2018, aponta que ocorreram no mundo 18 milhões de casos novos de câncer (17 milhões sem contar os casos de câncer de pele não melanoma) e 9,6 milhões de óbitos (9,5 milhões excluindo os cânceres de pele não melanoma). Para o Brasil, a estimativa para cada ano do triênio 2020-2022 aponta que ocorrerão 625 mil casos novos de câncer (450 mil, excluindo os casos de câncer de pele não melanoma) (Inca, 2019).

Durante a internação na UTI, é possível observar que, muitos casos concomitantes ao momento do diagnóstico, muitos pacientes encontram-se em um quadro clínico extremamente grave sem respostas às terapias de cura propostas. Nesse sentido, quando a assistência curativa não é mais indicada, os cuidados paliativos são aplicados, cujo objetivo é amenizar o sofrimento e promover o bem-estar do paciente e seus familiares (Casagrande et al, 2021; Oliveira, 2016).

Segundo a Organização mundial da saúde (OMS) cuidados paliativos consistem na assistência promovida por uma equipe multidisciplinar, que objetiva a melhoria da qualidade de vida do paciente e seus familiares, diante de uma doença que ameace a vida, por meio da prevenção e alívio do sofrimento, por meio de identificação precoce, avaliação impecável e tratamento de dor e demais sintomas físicos, sociais, psicológicos e espirituais (Inca, 2021).

Receber cuidados paliativos não significa que não haja mais nada a fazer por você ou pela pessoa que você ama. Isso simplesmente indica que o diagnóstico é de uma doença crônica grave, que ameaça a vida, e que uma equipe, juntamente com os profissionais especialistas na enfermidade, irá cuidar de quem está doente e daqueles que o cercam. Ou seja, "há muito a fazer" pelo paciente (ANCP, 2021). 
Em vista disso, reconhece-se a complexidade do paciente oncológico em cuidados paliativos, devido sua constante instabilidade no quadro clínico, o qual se faz necessário uma atenção maior do enfermeiro em realizar reavaliações frequentes de acordo com suas necessidades, de forma a promover o cuidado holístico, humanizado e individualizado. É importante que os enfermeiros obtenham conhecimento especializado para reconhecer as particularidades destes, a fim garantir a assistência adequada e com qualidade, bem como o bem-estar do paciente oncológico, os quais farão com que o mesmo tenha um fim de vida confortável e sem dor, dando atenção também à família mesmo após sua morte (Oliveira et al, 2020).

Dessa forma, os cuidados de enfermagem ao paciente oncológico internado em cuidados paliativos incluem cuidados de rotina da UTI, além de promover ao paciente o controle dos sinais e sintomas, principalmente o alívio da dor, pois em pacientes com doenças avançadas a prevalência de sintomas dolorosos é alta, no câncer a prevalência pode chegar a $90 \%$. Ademais, é imprescindível que a equipe forneça o conforto por meio de ações simples como o apoio psicológico, espiritual e emocional oferecido a família, a fim de estimulá-los a permanecer ao lado do paciente para superar os momentos difíceis de sua vida, bem como o de sua partida (Oliveira, 2016). Tais ações implicam além de uma conduta assistencial de qualidade, uma postura ética frente aos cuidados prestados, ao paciente e sua família.

Diante desta realidade dos cuidados paliativos que tem tão comum nos serviços de saúde oncológica, surgiu a inquietação de busca de conteúdos científicos que abordassem as questões éticas dos cuidados paliativos para a enfermagem no contexto da terapia intensiva. E não obstante à introdução do cuidado paliativo há décadas no ambiente da assistência à saúde, surgiu como questionamento se há lacunas nas discussões referentes nas produções científicas em saúde na abordagem ética em cuidados paliativos para a enfermagem, no contexto da terapia intensiva? Logo, objetivou realizar uma revisão integrativa das produções científicas e investigar sobre os aspectos éticos dos cuidados paliativos para a enfermagem na terapia intensiva.

\section{Metodologia}

Trata-se de uma Revisão Integrativa da Literatura (RIL) que proporciona a síntese de inúmeros artigos e contribui para condensar resultados de estudos referentes ao mesmo tema (Mendes, Silveira e Galvão, 2019). O presente artigo se ancora na abordagem qualitativa que ao se preocupar com um nível de realidade que não pode ser quantificado, trabalha com o universo de significados, motivações, aspirações, crenças, valores e atitudes, o que corresponde a um espaço mais profundo das relações (Minayo 2002).

A escolha da RIL ocorreu diante da busca de manuscritos que evidenciassem informações sobre o tema, a partir de estudos já publicados, pré-estabelecida da seguinte maneira: 1. Elaboração da questão de pesquisa, 2. Critérios de inclusão e exclusão, 3. Definição da amostragem, 4. Avaliação dos estudos incluídos, 5. Interpretação dos resultados e 6. Apresentação da síntese da RIL (Mendes, Silveira e Galvão, 2019).

A busca foi realizada por meio das bases de dados PUBMED, EMBASE, BVS/LILACS E CINAHL. Foram identificados 1035 artigos utilizando os termos "ética, enfermagem e cuidados paliativos" e realizado a leitura de seus resumos. Alguns manuscritos apresentaram conteúdos bem atuais em cuidados paliativos com enfoque ético para enfermagem. Entretanto, o foco era encontrar essa temática na terapia intensiva para atender ao objetivo proposto, logo acrescentamos o descritor "terapia intensiva" na busca inicial. Desta forma, dos 1035 manuscritos encontrados, 849 foram excluídos, restando 113 artigos para prosseguimento da análise (Figura 1).

Adotamos para o estudo como critérios de inclusão: artigos com resumo completo nos idiomas português, inglês e espanhol que abordassem a temática "ética, enfermagem, cuidados paliativos, terapia intensiva". Para os critérios de exclusão direcionamos aos artigos com resumos incompletos e sem o descritor UTI.

A questão da pesquisa foi elaborada com a utilização do acrônimo PICO, estratégia que orienta a construção da pergunta de pesquisa e da busca bibliográfica e permite ao pesquisador sistematizar o questionamento e localizar de modo 
acurado e rápido a melhor informação científica disponível (Araújo, 2020). Os elementos do acrônimo PICO ficaram organizados da seguinte forma:

$\mathbf{P}$ (paciente ou problema): aspectos éticos para enfermagem

I (intervenção): abordagem do contexto ético em cuidados paliativos

C (controle ou comparação): verificação de produções científicas que abordem os aspectos éticos para enfermagem em cuidados paliativos na terapia intensiva

$\mathbf{O}$ (desfechos): Não se aplica.

Dessa forma, surgiu a questão de pesquisa: quais as produções científicas que abordam os aspectos éticos para a enfermagem sobre os cuidados paliativos em terapia intensiva? A partir desta análise, foi realizada uma estratégia de busca bibliográfica, permitido alcançar a dimensão dos estudos sobre a temática na atualidade.

A busca seguiu duas etapas:

No primeiro momento foram utilizados os descritores: aspectos éticos, enfermagem e cuidados paliativos com os operadores booleanos OR e AND. Para cada base de dados, foram selecionados artigos nos idiomas português, inglês e espanhol num recorte temporal de dez anos. A justificativa da delimitação de tempo maior que cinco anos (que geralmente é preconizado) deve-se a possibilidade de encontrar mais artigos sobre a temática, considerando que o tema "cuidado paliativo" ainda é considerado uma abordagem recente, mas que está ganhando força em diversos os espaços da prática profissional, conduzindo a importantes discussões e reflexões.

No segundo instante foram usados os mesmos descritores com acréscimo do descritor "terapia intensiva", haja vista que é o cenário proposto para o estudo. Esses achados permitiram uma breve comparação dos manuscritos com e sem descritor UTI. Vale ressaltar que o assunto é pouco explorado dentro do âmbito da unidade de terapia intensiva, sendo assim, os estudos envolvendo a equipe de enfermagem dentro de questões éticas em cuidados paliativos é mais explorado em outros cenários.

No entanto, também foram analisados os artigos sem o descritor UTI, como forma de explorar seu conteúdo e comparar com os estudos que abordassem, de fato, a unidade terapia intensiva. Os achados foram categorizados da seguinte forma na Figura 1: 
Figura 1 - Fluxograma do estudo de revisão integrativa, conforme as recomendações PRISMA:
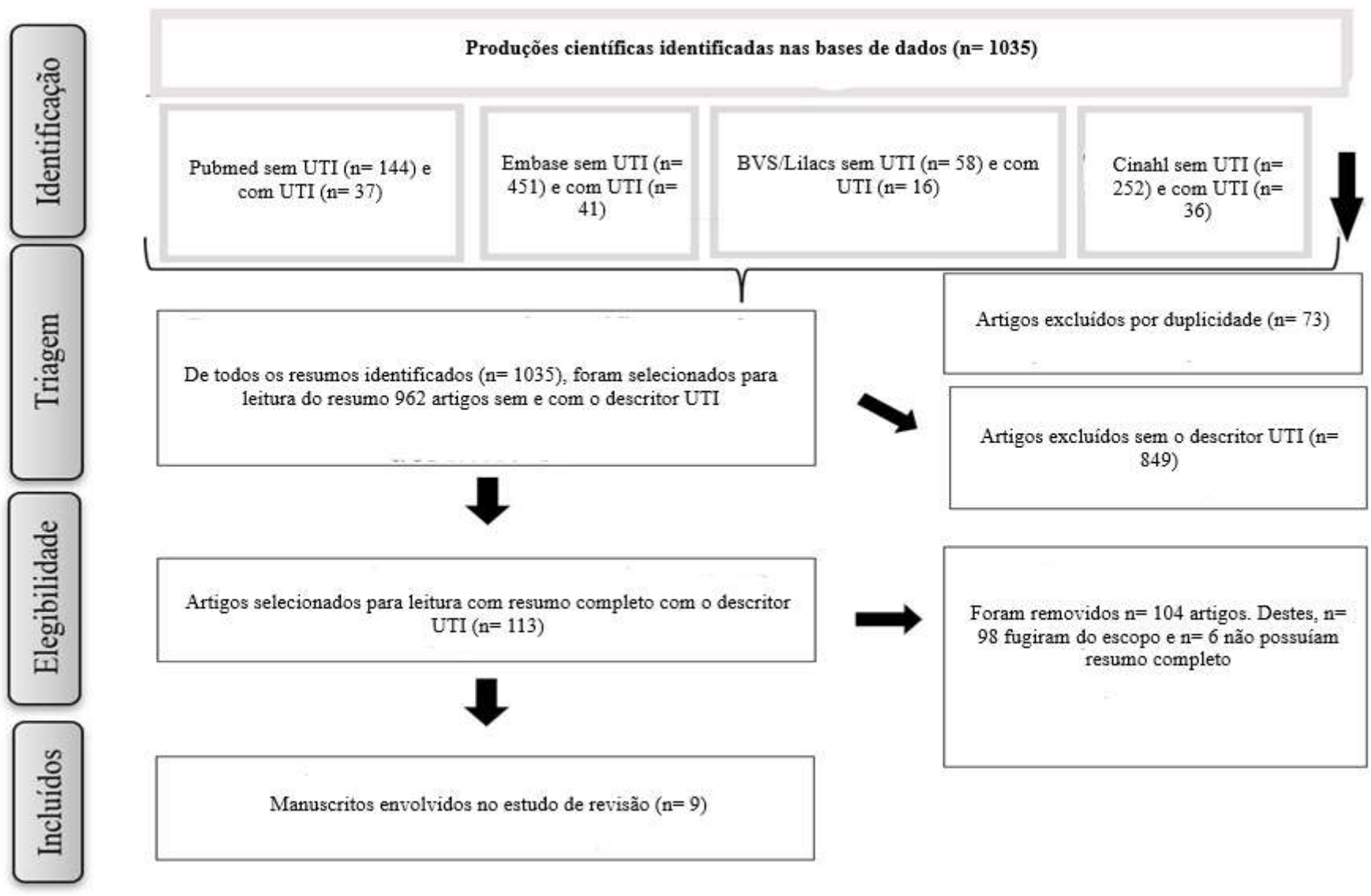

Manuscritos envolvidos no estudo de revisão $(n=9)$

Fonte: Dados elaborados pelos autores da pesquisa adaptado de Silva et al (2021).

Após análise, foram identificados 1035 artigos em todas as bases de dados descritas. Destes, foram eliminados os estudos duplicados (73 artigos) em ambos os casos (com e sem o descritor UTI), totalizando de fato, 962 artigos.

Ao incluir o descritor "terapia intensiva", foram achados 113 artigos.

Em contrapartida, desconsiderando o descritor "terapia intensiva” foram encontrados 849 artigos.

Consideramos os artigos com resumo completo nos idiomas inglês, português e espanhol. Para atender o proposto pela busca bibliográfica, foram selecionados os artigos com o descritor UTI com resumo completo nos idiomas descritos acima, resultando em 113 artigos. Destes, apenas 9 foram selecionados. Foram excluídos 104, sendo 98 por fugirem do seu escopo e 6 por não apresentarem resumo completo, apesar da tentativa de acesso do manuscrito completo pelo DOI e/ou ISSN.

Vale ressaltar que dos 9 artigos inclusos, apenas 8 referem-se aos últimos 5 anos. Ainda assim, consideramos 01 com mais de 5 anos de publicação por compreender que ao realizar o estudo da arte foi possível perceber que ainda é necessário estudos atuais sobre o assunto, haja vista que os cuidados paliativos e seus aspectos éticos estão cada vez mais presentes no contexto atual não só da oncologia, mas nos mais variados cenários.

Foi realizada leitura minuciosa dos resumos selecionados $(n=09)$ com o descritor UTI após essa primeira apreensão. Utilizamos suporte teórico para enriquecimento e sustentação das discussões.

Para o presente estudo foi dispensado o Termo de Consentimento Livre e esclarecido (TCLE) por se tratar de uma revisão integrativa sem envolvimento de seres humanos. 


\section{Resultados}

Foi possível perceber que $80 \%$ (7) dos artigos trata das questões da enfermagem relacionada aos cuidados paliativos e questões éticas, enquanto $20 \%$ (2) corresponde às indagações dos cuidados paliativos, envolvendo inclusive outros profissionais da saúde. E destes, 7 estavam disponíveis na língua inglesa e 2 na língua portuguesa.

Diante da análise o Quadro 1, a seguir, organiza os achados dos manuscritos por autores, ano de publicação, título, periódico publicado, objetivos, métodos e conclusão.

Quadro 1 - Síntese dos estudos incluídos na revisão segundo os autores, ano, título, objetivos, métodos e conclusão. Rio de Janeiro-RJ, 2021.

\begin{tabular}{|c|c|c|c|c|}
\hline & $\begin{array}{c}\text { ARTIGO } \\
\end{array}$ & OBJETIVOS & MÉTODOS & CONCLUSÃO \\
\hline 01 & $\begin{array}{c}\text { Altaker KW, } \\
\text { Howie-Esquivel J, Cataldo JK } \\
\text { (2018). } \\
\text { Relationships Among Palliative } \\
\text { Care, Ethical Climate, } \\
\text { Empowerment, and Moral Distress } \\
\text { in Intensive Care Unit Nurses. } \\
\text { AJCC-American journal critical care }\end{array}$ & $\begin{array}{l}\text { To evaluate } \\
\text { relationships among } \\
\text { moral distress, } \\
\text { empowerment, ethical } \\
\text { climate, and access to } \\
\text { palliative care in the } \\
\text { intensive care unit. }\end{array}$ & $\begin{array}{l}\text { Intensive care unit nurses in a national } \\
\text { database were recruited to complete an } \\
\text { online survey based on the Moral } \\
\text { Distress Scale-Revised, Psychological } \\
\text { Empowerment Index, Hospital Ethical } \\
\text { Climate Survey, and a palliative care } \\
\text { delivery questionnaire. Descriptive, } \\
\text { correlational, and regression analyses } \\
\text { were performed. }\end{array}$ & $\begin{array}{l}\text { Poor ethical climate, unintegrated } \\
\text { palliative care teams, and nurse } \\
\text { empowerment are associated with } \\
\text { increased moral distress. The } \\
\text { findings highlight the need to } \\
\text { promote palliative care education } \\
\text { and palliative care teams that are } \\
\text { well integrated into intensive care } \\
\text { units. }\end{array}$ \\
\hline 02 & $\begin{array}{l}\text { D’Arco C, Maluf CM, Ferrari } \\
\text { LVBC, Priel LLP (2016) } \\
\text { Obstinação terapêutica sob o } \\
\text { referencial bioético } \\
\text { da vulnerabilidade na prática da } \\
\text { enfermagem. } \\
\text { O mundo da saúde São Paulo }\end{array}$ & $\begin{array}{l}\text { O objetivo da presente } \\
\text { pesquisa foi o de } \\
\text { compreender a } \\
\text { percepção do } \\
\text { enfermeiro de uti sobre } \\
\text { obstinação terapêutica, } \\
\text { à luz do referencial } \\
\text { bioético da } \\
\text { vulnerabilidade. }\end{array}$ & $\begin{array}{l}\text { Utilizou-se a pesquisa descritiva, análise } \\
\text { do conteúdo de Bardin, com quatorze } \\
\text { estudantes pós-graduandos do curso de } \\
\text { especialização de uma instituição de } \\
\text { ensino superior privada que atuavam em } \\
\text { uti, há mais de um ano através de } \\
\text { entrevistas. }\end{array}$ & $\begin{array}{l}\text { Evidenciou-se a necessidade de } \\
\text { inclusão dos aspectos bioéticos na } \\
\text { formação do enfermeiro, pois } \\
\text { identifica-se que estes não } \\
\text { conhecem o conceito de obstinação } \\
\text { terapêutica, porém reconhecem o } \\
\text { prolongamento do processo de } \\
\text { morte como negativo. } \\
\text { principalmente quando estão } \\
\text { envolvidos jovens, ainda sim, } \\
\text { reconhecem seu papel na } \\
\text { terminalidade. }\end{array}$ \\
\hline 03 & $\begin{array}{l}\text { Ganz FD, Sapir B (2019) } \\
\text { Nurses' perceptions of intensive care } \\
\text { unit palliative care at end of life. } \\
\text { Nurs Crit Care }\end{array}$ & $\begin{array}{l}\text { To describe ICU } \\
\text { nurses' perceptions of } \\
\text { quality palliative end- } \\
\text { of-life care, barrier } \\
\text { intensity and } \\
\text { frequency to palliative } \\
\text { care and their } \\
\text { association with one } \\
\text { another. This was a } \\
\text { descriptive, } \\
\text { correlational, cross- } \\
\text { sectional design. }\end{array}$ & $\begin{array}{l}\text { A convenience sample of } 126 \text { ICU } \\
\text { nurses from two hospitals in Israel was } \\
\text { recruited for the study. Participants } \\
\text { completed three pencil-and-paper } \\
\text { questionnaires (a personal } \\
\text { characteristics questionnaire, the Quality } \\
\text { of Palliative Care in the ICU and a } \\
\text { revised Survey of Oncology Nurses' } \\
\text { Perceptions of End-of-Life Care). } \\
\text { Respondents were recruited during staff } \\
\text { meetings or while on duty in the ICU. } \\
\text { Ethical approval was obtained for the } \\
\text { study from participating hospitals. }\end{array}$ & $\begin{array}{l}\text { ICU nurses perceived the quality } \\
\text { of palliative care at the end of life } \\
\text { as moderate despite reports of } \\
\text { moderate barrier levels. The } \\
\text { frequency of barriers was weakly } \\
\text { associated with quality palliative } \\
\text { end-of-life care. However, barrier } \\
\text { intensity did not correlate with } \\
\text { quality palliative end-of-life care at } \\
\text { a statistically significant level. } \\
\text { Further research that investigates } \\
\text { other factors associated with } \\
\text { quality ICU palliative care is } \\
\text { recommended. relevance to } \\
\text { clinical practice: Barriers to } \\
\text { palliative care are still common in } \\
\text { the ICU. Increased training and } \\
\text { education are recommended to } \\
\text { decrease barriers and improve the } \\
\text { quality of ICU palliative care. }\end{array}$ \\
\hline 04 & $\begin{array}{c}\text { Browning ED, } \\
\text { Cruz JS (2018) } \\
\text { Reflective Debriefing: A Social } \\
\text { Work Intervention Addressing } \\
\text { Moral Distress among ICU Nurses. } \\
\text { Journal Soc Work End Life Palliat } \\
\text { Care }\end{array}$ & $\begin{array}{l}\text { Health-care workers } \\
\text { may experience moral } \\
\text { distress when they are } \\
\text { unable to act as they } \\
\text { believe is ethically } \\
\text { appropriate in their } \\
\text { clinical work. A social } \\
\text { worker-facilitated } \\
\text { protocol called } \\
\text { Reflective Debriefing } \\
\text { was developed and } \\
\text { tested for alleviating } \\
\text { moral distress through } \\
\text { regular debriefings } \\
\text { with nursing staff on }\end{array}$ & $\begin{array}{l}\text { Forty-two ICU nurses completed a } \\
\text { Moral Distress Scale-Revised (MDS-R) } \\
\text { at the beginning and end of a 6-month } \\
\text { period, during which time regular } \\
\text { debriefings were offered. The overall } \\
\text { level of moral distress on the ICU } \\
\text { surveyed was found to be low to } \\
\text { moderate. }\end{array}$ & $\begin{array}{l}\text { The response to the intervention } \\
\text { emphasized the importance of } \\
\text { interprofessional collaboration to } \\
\text { successfully combat moral distress } \\
\text { among health-care workers and } \\
\text { may protect them from burnout, } \\
\text { detachment, and even quitting their } \\
\text { profession. }\end{array}$ \\
\hline
\end{tabular}




\begin{tabular}{|c|c|c|c|c|}
\hline & & $\begin{array}{l}\text { an intensive care unit } \\
\text { (ICU). }\end{array}$ & & \\
\hline 05 & $\begin{array}{c}\text { Cavalcanti ÍMC, Oliveira LO, } \\
\text { Macêdo LC, Leal MHC, Morimura } \\
\text { MCR, Gomes ET (2019) } \\
\text { Princípios dos cuidados paliativos } \\
\text { em terapia intensiva na perspectiva } \\
\text { dos enfermeiros. } \\
\text { Revista Cuidarte }\end{array}$ & $\begin{array}{l}\text { Avaliar a percepção } \\
\text { dos enfermeiros } \\
\text { intensivistas acerca da } \\
\text { adesão aos princípios } \\
\text { dos cuidados } \\
\text { paliativos na sua } \\
\text { prática assistencial. }\end{array}$ & $\begin{array}{l}\text { Estudo correlacional descritivo, de corte } \\
\text { seccional, realizado com } 104 \\
\text { enfermeiros de doze unidades de terapia } \\
\text { intensiva em cinco hospitais de uma } \\
\text { capital do Nordeste do Brasil, entre } \\
\text { janeiro e dezembro de } 2017 \text {. }\end{array}$ & $\begin{array}{l}\text { Os dois princípios com menores } \\
\text { escores referem-se a dilemas éticos } \\
\text { importantes para os profissionais e } \\
\text { que devem ser amplamente } \\
\text { discutidos. }\end{array}$ \\
\hline 06 & $\begin{array}{c}\text { Lin CC (2017) } \\
\text { Knowledge and attitudes towards } \\
\text { advances directives and quality of } \\
\text { palliative care in intensive care units: } \\
\text { A perspective comparison between } \\
\text { physicians and registered nurses. } \\
\text { Supportive Care in Cancer }\end{array}$ & $\begin{array}{l}\text { To understand the } \\
\text { knowledge and } \\
\text { attitudes of physicians } \\
\text { and nurses toward } \\
\text { advance directives and } \\
\text { their viewpoints on the } \\
\text { quality of palliative } \\
\text { care in an ICU. }\end{array}$ & $\begin{array}{l}\text { A cross-sectional study. } 56 \text { physicians } \\
\text { and } 120 \text { nurses in ICU in Taiwan. }\end{array}$ & $\begin{array}{l}\text { Providing ICU clinicians with } \\
\text { emotional and educational support } \\
\text { regarding end-of-life care and } \\
\text { encouraging nurses to participate } \\
\text { in making end-of-life medical } \\
\text { decisions may improve the quality } \\
\text { of palliative care in ICU. }\end{array}$ \\
\hline 07 & $\begin{array}{l}\text { Gallagher A et al. (2015). } \\
\text { Negotiated reorienting: a grounded } \\
\text { theory of nurses' end-of-life } \\
\text { decision-making in the intensive } \\
\text { care unit. } \\
\text { Int J Nurs Stud }\end{array}$ & $\begin{array}{l}\text { To understand nurses' } \\
\text { EoL decision-making } \\
\text { practices in ICUs in } \\
\text { different cultural } \\
\text { contexts. }\end{array}$ & $\begin{array}{l}\text { We collected and analysed qualitative } \\
\text { data using Grounded Theory. Interviews } \\
\text { were conducted with experienced ICU } \\
\text { nurses in university or hospital premises } \\
\text { in five countries: Brazil, England, } \\
\text { Germany, Ireland and Palestine. } \\
\text { PARTICIPANTS: Semi-structured } \\
\text { interviews were conducted with } 51 \\
\text { nurses ( } 10 \text { in Brazil, } 9 \text { in England, } 10 \text { in } \\
\text { Germany, } 10 \text { in Ireland and } 12 \text { nurses in } \\
\text { Palestine). They were purposefully and } \\
\text { theoretically selected to include nurses } \\
\text { having a variety of characteristics and } \\
\text { experiences concerning end-of-life } \\
\text { (EoL) decision-making. }\end{array}$ & $\begin{array}{l}\text { There was consensus regarding the } \\
\text { core concept and core practices } \\
\text { employed by nurses in the ICUs in } \\
\text { the five countries. However, there } \\
\text { were some discernible differences } \\
\text { regarding the power dynamics in } \\
\text { nurse-doctor relationships, } \\
\text { particularly in relation to the } \\
\text { cultural perspectives on death and } \\
\text { dying and in the development of } \\
\text { palliative care. The research } \\
\text { suggests the need for culturally } \\
\text { sensitive ethics education and } \\
\text { bereavement support in different } \\
\text { cultural contexts. }\end{array}$ \\
\hline 08 & $\begin{array}{l}\text { Hagger V, Ellis C, Strumidlo L } \\
\qquad(2016) . \\
\text { Legal and ethical issues in neonatal } \\
\text { nursing: a case study. } \\
\text { Nursing Standard }\end{array}$ & $\begin{array}{l}\text { This article discusses } \\
\text { the hypothetical case } \\
\text { of Jack, a two-day-old } \\
\text { infant diagnosed with } \\
\text { trisomy } 13 \\
\text { (syndrome), a life- } \\
\text { limiting condition. }\end{array}$ & $\begin{array}{l}\text { The article also discusses the neonatal } \\
\text { nurse's role, with reference to local and } \\
\text { national guidelines. }\end{array}$ & $\begin{array}{l}\text { The legal and ethical perspectives } \\
\text { of withholding life-sustaining } \\
\text { treatment in infants and children } \\
\text { will be explored through the } \\
\text { application of ethical frameworks, } \\
\text { as well as statute and case law } \\
\text { relevant to children and adolescent } \\
\text { nursing. }\end{array}$ \\
\hline 09 & $\begin{array}{c}\text { Harorani M et al (2019) } \\
\text { Moral distress and self-efficacy } \\
\text { among nurses working in critical } \\
\text { care unit in Iran-an analytical study. } \\
\text { Journal of Clinical and Diagnostic } \\
\text { Research }\end{array}$ & $\begin{array}{l}\text { To determine the } \\
\text { average frequency and } \\
\text { intensity of moral } \\
\text { distress and its } \\
\text { relationship with self- } \\
\text { efficacy in nurses } \\
\text { working in Critical } \\
\text { care units. }\end{array}$ & $\begin{array}{l}\text { This study was analytic one, conducted } \\
\text { on } 300 \text { nurses working in critical care } \\
\text { unit selected through simple random } \\
\text { sampling method. The data were } \\
\text { collected using demographic } \\
\text { characteristics questionnaire, Corley's } \\
\text { moral distress scale, and Riggs et al., } \\
\text { self-efficacy beliefs questionnaire. The } \\
\text { data were analysed using descriptive } \\
\text { statistics and Pearson's correlation } \\
\text { coefficient. }\end{array}$ & $\begin{array}{l}\text { This study showed that there is a } \\
\text { significant negative relationship } \\
\text { between moral distress and the } \\
\text { level of perceived self-efficacy, } \\
\text { i.e., by an increase in moral } \\
\text { distress, the level of perceived self- } \\
\text { efficiency in nurses decreases. }\end{array}$ \\
\hline
\end{tabular}

Fonte: Elaborado pelos autores do estudo (2021).

Sequencialmente à inspeção dos documentos, o estudo possibilitou a divisão dos artigos e a construção de três categorias:

A- $\quad$ Sofrimento moral diante da situação de morte;

B- Compressão dos enfermeiros sobre cuidados paliativos;

C- Dilemas éticos em cuidados paliativos. 


\section{Discussão}

\section{A-Sofrimento moral diante da situação de morte}

Foi possível identificar que as produções científicas abordam os cuidados paliativos de forma muito interessante quando se trata de sofrimento moral dos profissionais, enfatizando a preocupação dos enfermeiros diante de questões éticas e do que consideram como condutas corretas. Enfermeiros de unidade de terapia intensiva vivenciam sofrimento moral quando se sentem incapazes de prestar cuidados eticamente adequados aos pacientes. O sofrimento moral está associado ao esgotamento da enfermagem e à evitação do atendimento ao paciente e correlacionado ao empoderamento destes profissionais, pois enfermeiros que relataram acesso aos cuidados paliativos tiveram um escore de avaliação mais elevado de sofrimento moral. É mister pensarmos que os conhecimentos destes profissionais podem levá-los a muitas indagações/ questionamento sobre condutas acerca da paliação dos pacientes.

Na abordagem do artigo 01 é importante ressaltar que etnia, tamanho da unidade de atuação e acesso aos cuidados paliativos explicaram as variações dos escores de sofrimento moral. Porém com clima ético pobre, equipes de cuidados paliativos não integrados e capacitação da equipe está relacionado ao aumento desse sofrimento, logo o estudo aponta para promoção de educação em cuidados paliativos e integração das equipes de cuidados paliativos às unidades de terapia intensiva (Altaker et al., 2018). Ratificando as questões de sofrimento moral, o manuscrito 04 corrobora ao dizer que profissionais de saúde podem passar por sofrimento moral quando são incapazes de agir conforme acreditam se eticamente apropriado em seu trabalho clínico. Neste mesmo estudo foi proposto um protocolo denominado "ambiente reflexivo" para aliviar o sofrimento moral por meio de ambientess regulares com a equipe de enfermagem em uma UTI e as três principais situações de sofrimento morais mais frequentes relatadas pela maioria dos enfermeiros deste estudo estavam relacionadas à prestação de cuidados não benéficos na UTI como prolongamento da vida às custas do sofrimento do paciente, medidas invasivas e sedação ineficiente.

Os participantes relataram obter mais benefícios ao se sentirem capacitados para confrontar construtivamente outros membros da equipe sobre como dizer a verdade ao dar um prognóstico. A resposta geral dos enfermeiros à intervenção foi positiva, com totalidade dos participantes solicitando a continuação das sessões de um 'ambiente reflexivo mensalmente" ou conforme a necessidade. A resposta à intervenção enfatizou a importância da colaboração interprofissional para combater com sucesso o sofrimento moral entre os profissionais de saúde e pode proteger eles do esgotamento, do distanciamento e até do abandono da profissão (Browning \& Cruz, 2016).

O manuscrito 09 trata o sofrimento moral como um dos principais problemas que podem afetar adversamente o sistema de saúde e é definido como desconforto na paz mental e ocorre em decorrência da omissão de ações morais adequadas, apesar do conhecimento. Há um nível moderado de sofrimento moral entre enfermeiros de unidades de terapia intensiva, tanto em frequência quanto em intensidade. Além disso, os resultados mostraram que diferentes enfermarias afetam a frequência e a intensidade do sofrimento moral em enfermeiras de forma diferente, uma vez que as enfermeiras de UTI experimentaram maior sofrimento moral do que as enfermeiras de outros departamentos. Existe uma relação negativa significativa entre o sofrimento moral e o nível de autoeficácia percebida, ou seja, ao aumentar o sofrimento moral, o nível de autoeficácia percebida nos enfermeiros diminui (Harorani, 2019).

\section{B-Compressão dos enfermeiros sobre cuidados paliativos}

O artigo 03 enfatiza a questão dos conhecimentos dos enfermeiros acerca dos cuidados paliativos. Discorre que é necessário o conhecimento e que a partir deste o profissional mostra-se questionador e isso afeta e repercuti positivamente na qualidade dos cuidados prestados, quanto contribui com o sofrimento quando as condutas são desencontradas, levando a um sofrimento moral, por exemplo, como é abordado na categoria anterior. 
Enfermeiros de UTI percebem a qualidade dos cuidados paliativos no final de vida como moderada, apesar dos relatos de níveis moderados de barreira. A frequência das barreiras foi fracamente associada a cuidados paliativos de qualidade no fim de vida. Recomendam-se mais pesquisas que investiguem outros fatores associados aos cuidados paliativos de qualidade. As barreiras aos cuidados paliativos ainda são comuns na UTI, mais treinamento e educação são recomendados para diminuir barreiras e melhorar a qualidade dos cuidados paliativos na UTI (Ganz \& Sapir, 2020).

Nos artigos 05 e 06 inferem a qualidade dos processos paliativos e da relevância de todos os processos envolvidos. Compreender e aplicar os cuidados paliativos na prática profissional em cuidados intensivos é de suma importância. Foi atribuído um valor para a relevância e importância de cada princípio na sua prática assistencial junto a pacientes em estágio terminal de doença. Os princípios que se mostraram mais relevantes à prática assistencial dos enfermeiros entrevistados foram: aliviar a dor e outros sintomas associados; garantir a qualidade da vida e do morrer; priorizar sempre o melhor interesse do paciente e, respeitar a autonomia do doente e seus representantes legais. Já os que receberam menores escores, foram afirmar a vida e considerar a morte como um processo normal da vida e avaliar o custo-benefício a cada atitude médica assumida. Os enfermeiros mostraram reconhecer elevada relevância/importância para os princípios de cuidados paliativos em terapia intensiva. Os dois princípios com menores escores referem-se a dilemas éticos importantes para os profissionais e que devem ser amplamente discutidos (Cavalcanti et al, 2019).

O conhecimento e as atitudes do pessoal médico em relação às diretrizes antecipadas (DAs), que é parte do processo dos cuidados paliativos, podem exercer influência em sua comunicação com os pacientes e na qualidade do cuidado ao paciente no final da vida. A pontuação para o conhecimento do médico sobre as diretrizes antecipadas foi maior do que a dos enfermeiros. Os médicos e enfermeiras adotaram uma atitude positiva em relação à participação na tomada de decisões médicas para o fim da vida. O escore médio da qualidade dos cuidados paliativos dos médicos foi de 6,59, significativamente inferior ao da qualidade dos cuidados paliativos dos enfermeiros. Além disso, 96,7\% dos enfermeiros acham que devem participar da tomada de decisão do fim da vida, enquanto cerca de 83,9\% dos médicos e 59,5\% dos enfermeiros concordaram em obter consenso quando a decisão do fim da vida foi tomada.

Os médicos e enfermeiras exibiram desempenho insatisfatório nos domínios do apoio espiritual de pacientes e familiares e no domínio do apoio hospitalar, o fornecimento de apoio emocional para a equipe médica que cuida de pacientes moribundos e a continuidade dos cuidados. Os fatores preditivos para a autoavaliação da qualidade dos enfermeiros para os cuidados paliativos no fim da vida foram à atitude em relação à tomada de decisões médicas sobre o fim da vida e a participação do enfermeiro na tomada de decisões médicas sobre o fim da vida. Fornecer aos médicos da UTI apoio emocional e educacional em relação aos cuidados no fim da vida e encorajar os enfermeiros a participarem na tomada de decisões médicas sobre o fim da vida pode melhorar a qualidade dos cuidados paliativos na UTI (Lin, 2017; Desanoski, 2019).

\section{C-Dilema ético em cuidados paliativos}

Já nos artigos 02, 07 e 08 a análise passa pelo dilema ético nesse cuidado e convoca para que possamos discutir essas questões que estão intimamente ligadas aos cuidados paliativos e evoca a tecnologia nesse processo. E quando se trata de UTI é muito comum a tecnologia dura atravessando as demandas dos cuidados paliativos A tecnologia desencadeia dilemas bioéticos, entre eles a obstinação terapêutica. E frente a esses dilemas enfermeiros priorizam avanços tecnológicos aos saberes reflexivos. Muitas vezes o desconhecimento do conceito sobre cuidados paliativos gera passividade diante das decisões promovendo dificuldade de enfrentar a terminalidade impactando negativamente na obstinação terapêutica, assim como seu desconhecimento sobre obstinação terapêutica influencia na vulnerabilidade do enfermeiro ao vivenciar tal situação, que provavelmente não foi preparado durante a graduação e pós-graduação, principalmente quando os envolvidos são jovens ou crianças. 
Também estão despreparados a reconhecer quando a recuperação do paciente está em detrimento à tecnologia, necessitando consenso multiprofissional. Enfermeiros reconhecem a necessidade de controle da dor, diminuição do sofrimento e promoção da dignidade. Há necessidade de inclusão dos aspectos bioéticos na formação do enfermeiro, pois identifica-se que estes não conhecem o conceito de obstinação terapêutica, porém reconhecem o prolongamento do processo de morte como negativo (D’Arco et al, 2016). As questões éticas surgem quando é necessário tomar decisões com relação à suspensão e recusa do tratamento de suporte à vida e à mudança para o conforto e os cuidados paliativos. Essas questões são particularmente desafiadoras para os enfermeiros quando há vários graus de incerteza quanto ao prognóstico. Pouco se sabe sobre a prática de tomada de decisão de fim de vida das enfermeiras entre as culturas.

A análise comparativa dos dados dentro e entre os dados gerados pelas diferentes equipes de pesquisa permitiu aos pesquisadores desenvolver uma compreensão mais profunda das práticas de tomada de decisão eol na UTI. A categoria central que emergiu foi reorientação negociada, embora os enfermeiros não tomem as decisões finais, eles se envolvem em duas práticas principais busca de consenso envolvendo persuasão, orientação de informações e habilitação por voz; e retenção emocional criando espaço-tempo e dando conforto. Houve consenso sobre o conceito central e as práticas básicas empregadas por enfermeiros nas utis dos cinco países. No entanto, havia algumas diferenças perceptíveis em relação à dinâmica de poder nas relações enfermeiro-médico, principalmente em relação às perspectivas culturais sobre a morte e o morrer e no desenvolvimento dos cuidados paliativos, que sugere a necessidade de educação ética culturalmente sensível e apoio ao luto em diferentes contextos culturais (Gallagher et al, 2015; Silva et al, 2018).

Enfermeiras nas mais variadas especialidades enfrentam regularmente dilemas legais e éticos complexos. Decisões legais e éticas de suspender o tratamento de suporte de vida devem ser exploradas por meio da aplicação de estruturas éticas, bem como estatutos e jurisprudência relevantes para a enfermagem e paciente assistido (Hagger et al., 2016).

\section{Conclusão}

Apesar dos avanços nas discussões e condutas acerca dos cuidados paliativos na atualidade, ainda enfrentamos diversos desafios nos espaços assistenciais. Essas dificuldades refletem numa atuação que perpassa a multidisciplinaridade, mas convoca todos os profissionais envolvidos para uma ação tanto interdisciplinar, quando transdisciplinar em prol do paciente e sua família.

Não é incomum assistir às angústias dos profissionais diante da introdução dos cuidados paliativos na terapia intensiva, visto como um local de cura e do restabelecimento do paciente. Essa necessidade de "cura" e resolutividade pode gerar falsas expectativas, além de serem extremamente danosa na relação paciente-profissional-família.

É urgente pensarmos e se necessário repensarmos as condutas diante do paciente e traçar estratégias para a abordagem e prática dos cuidados paliativos em UTI, haja vista a importância da introdução deste cuidado tão essencial desde o diagnóstico. Ressalta-se também a importância de uma comunicação efetiva entre os profissionais da equipe para diminuir a distância entre as condutas, assim como os dilemas éticos que atravessam esse processo.

Não obstante ao progresso das produções científicas sobre questões éticas dos cuidados paliativos em terapia intensiva, é de suma importância que a temática seja amplamente discutida para que os profissionais implicados, ao se depararem com o paciente em cuidados paliativos na terapia intensiva possa ter condutas mais assertivas e comunicação mais inclusiva/participativa de todos os profissionais na tomada de decisões.

Espera-se que o presente estudo contribua com os avanços no setor saúde e na reflexão acerca da importância da discussão dos aspectos éticos para a enfermagem em cuidados paliativos. E que esta temática possa fazer parte não só do cotidiano da prática profissional, mas que possa fazer parte do processo de sua formação acadêmica. 


\section{Referências}

Agência Nacional de Cuidados Paliativos (ANCP) (2021). O que são cuidados paliativos? Recuperado de https://paliativo.org.br/cuidados-paliativos/o-quesao/

Altaker, K. W., Howie-Esquivel. J. \& Cataldo, J. K. (2018). Relationships Among Palliative Care, Ethical Climate, Empowerment, and Moral Distress in Intensive Care Unit Nurses, AJCC-American journal critical care. 27(4), 295-302. doi.org/ 10.4037/ajcc2018252.

Araújo, W. C. O. (2020). Recuperação da informação em saúde: construção, modelos e estratégias, ConCl: Conv. Ciênc.Inform. 3(2), 100-34. doi.org/10.33467/conci.v3i2.13447

Brasil, Ministério da Saúde. (2020). Resolução $N^{\mathbf{o}}$ 2.271/2020 que define as unidades de terapia intensiva, conforme complexidade, nível de cuidado, responsabilidade técnica, habilitações e atribuições para seu funcionamento. Recuperado de https://www.gov.br

Browning, E. D. \& Cruz, J. S. (2018). Reflective Debriefing: A Social Work Intervention Addressing Moral Distress among ICU Nurses, Journal of Social Work in End-of-Life \& Palliative Care. 14(1), 44-72. doi .org/10.1080/15524256.2018.1437588

Casagrande, E. F., Araújo, B. N., Dezordi, C. C. N. \& Fracaro, L. D (2021). Assistência de enfermagem ao paciente em cuidados paliativos acometido por mieloma múltiplo internado em unidade de terapia intensiva. XXI Jornada de Extensão ciências da saúde da Universidade Regional do Noroeste do Estado do RS. 7(7), 1-5. Recuperado de https://publicacoeseventos.unijui.edu.br/index.php/salaoconhecimento/article/view/20699/19411.

Cavalcanti, Í. M. C., Oliveira, L. O., Macêdo, L. C., Leal, M. H. C., Morimura, M. C. R. \& Gomes, E. T. (2019). Princípios dos cuidados paliativos em terapia intensiva na perspectiva dos enfermeiros. Revista Cuidarte. 10(1), e555. doi.org/10.15649/cuidarte. v10i1.555

Cavalcanti, N. A, Pinto, K. D. C. \& Maia, E. M. C. (2019). Perfil de pacientes adultos em Unidade de Terapia Intensiva do nordeste brasileiro, Revista: saúde e sociedade. 4(2), 1113-25. doi.org/10.28998/rpss. v4i2.6455

Conselho Federal de Medicina (CFM). (2020). Regulamenta a Resolução n ${ }^{\circ}$ 2.271/2020, que estabelece os critérios para funcionamento de Unidades de Terapia Intensiva e Unidades de Cuidados intermediários no Brasil (15p). Recuperado de http://www.portalmedico.org.br

D’Arco, C., Maluf, C. M., Ferrari, L. V. B. C. \& Priel, L. L. P (2016). Obstinação terapêutica sob o referencial bioético da vulnerabilidade na prática da enfermagem. Mundo da saúde São Paulo. 40(3), 382-389.

Desanoski, P. B. C., Shibukawa, B. M. C., Rissi, G. P., Silva, J. D. D. \& Higarashi, I. H. (2019). Cuidados paliativos: conhecimento de enfermeiros e aplicabilidade no âmbito hospitalar. Publ. UEPG Ci. Biol. Saúde, Ponta Grossa. 25(1), 28-36. doi.org/10.5212/Publ.Biologicas. v.25i1.0003

Gallagher, A., Bousso, R. S., McCarthy, J., Kohlen, H., Andrews, T., Peganini, M. C. \& Abu-El-Noor, N. I. (2015). Negotiated reorienting: a grounded theory of nurses' end-of-life decision-making in the intensive care unit. Int J Nurs Stud. 52(4), 794-803. doi.org/10.1016/j.ijnurstu.2014.12.003

Ganz, F. D \& Sapir, B. (2020). Sapir B. Nurses' perceptions of intensive care unit palliative care at end of life. Nurs Crit Care. 05-24(3), 141-148.

Hagger, V., Ellis, C. \& Strumidlo, L. (2016). Legal and ethical issues in neonatal nursing: a case study. Nursing Standard. 29-30(44), 48-53. doi.org/10.7748/ns. 2016.e10343

Harorani1, M., Golitaleb, M., Davodabady, F., Zahedi., Houshmand, S. M., Mousavi, S. S. \& Yousefi, M. S. (2019). Moral distress and self-efficacy among nurses working in critical care unit in Iran-an analytical study. Journal of Clinical and Diagnostic Research. 13(11), 6-9. doi.org/10.7860/JCDR/2019/41053.13266

Instituto Nacional de Câncer José Alencar Gomes da Silva (2020). Estimativa 2020: incidência de câncer no Brasil -Rio de Janeiro-RJ: INCA. Recuperado de https://www.inca.gov.br/estimativa

Instituto Nacional de Câncer José Alencar Gomes da Silva (2021). Cuidados paliativos segundo a Organização mundial da saúde (OMS)-Rio de Janeiro-RJ: INCA. Recuperado de https://www.inca.gov.br/search/conteudo/cuidados\%20paliativos

Lin, C. C. (2017). Knowledge and attitudes towards advances directives and quality of palliative care in intensive care units: a perspective comparison between physicians and registered nurses. Supportive Care in Cancer. 25, 21-266. doi.org/10.1007/s00520-017-3704-x

Mendes, K. D. S., Silveira, R. C. C. P. \& Galvão, C. M. (2019). Uso de gerenciador de referências bibliográficas na seleção dos estudos primários em Revisão Integrativa, Texto \& Contexto Enfermagem. 28, e20170204. doi.org/10.1590/1980-265X-TCE-2017-0204

Minayo, M. C. S. Pesquisa social: teoria, método e criatividade (2002). Ciência, técnica e arte: o desafio de pesquisa social. Petrópolis: Vozes.

Oliveira, T. F. (2016). Intervenções de enfermagem aos pacientes oncológicos em cuidados paliativos internados em uma unidade de terapia intensiva. Revista Eletrônica Gestão e Saúde. 7(1), 343-355.

Oliveira, T. S., Martins, B. C. T., Rocha, M. E., Gomes, N. S. \& Aires, V. G. S. (2020). Sistematização da Assistência de Enfermagem: análise da produção científica em oncologia-revisão integrativa, Revista Brazilian Journal of Development. 6(2), 9541-9555. doi.org/10.34117/bjdv6n2-314

Silva, A. C. A., Silva, M. L., Oliveira, M. R., Sales, D. R., Santos, A. C. C. \& Silva, R. A. (2018). Reflexões sobre o fim da vida: ética em cuidado. Revista Saúde. 12(1). Recuperado de http://revistas.ung.br/index.php/saude/article/view/3583

Silva, T. G., Oliveira, K. M. L., Morais, S. C. R. V., Perreli, J. G. A., Sousa, S. M. A. \& Linhares, F. M. P. (2021). Disfunção sexual em mulheres com câncer do colo do útero submetidas à radioterapia: análise de conceito, Revista da Escola Anna Nery. 25(4), 20200404. doi.org/10.1590/2177-9465-EAN-2020-0404 